\title{
Increased interest in acute pulmonary embolism in Italy during the COVID-19 pandemic: a google trends-based analysis
}

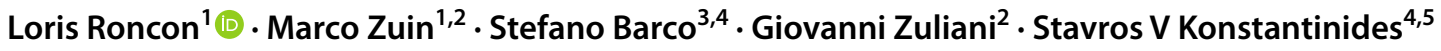

Accepted: 5 November 2020 / Published online: 11 November 2020

(c) Springer Science+Business Media, LLC, part of Springer Nature 2020

\section{To the Editor,}

Acute pulmonary embolism (PE) represents a frequent complication of COVID-19 infection among hospitalized patients [1]. Doubtless, social media have played a pivotal role in spreading awareness and knowledge about the COVID-19 pandemic, especially during the lockdown periods in different countries [2, 3]. In this regard, social media have widely presented acute PE as a complication of COVID-19 infection worldwide with unavoidable repercussion. However, no data regarding the trend of interest among general population on acute PE during the COVID-19 pandemic in Italy has been yet provided. In the present manuscript, we analysed Internet search data using Google Trends (GT) (https://trends.google.com) (Google, Mountain View, California, USA), to estimate the awareness and interest of the Italian population in acute PE during the acute phase of the pandemic as well as during the period characterized by a reduction of restrictive measures and the "second-phase" of the pandemic.

Loris Roncon and Marco Zuin have equally contributed to the manuscript

Loris Roncon

lorisroncon@gmail.com

1 Department of Cardiology, Santa Maria della Misericordia Hospital, Rovigo General Hospital Viale tre Martiri, 45100 Rovigo, Italy

2 Department of Morphology, Surgery and Experimental Medicine, University of Ferrara, Ferrara, Italy

3 Center for Thrombosis and Hemostasis (CTH), University Medical Center of the Johannes Gutenberg University, Mainz, Germany

4 Clinic of Angiology, University Hospital Zurich, Zurich, Switzerland

5 Department of Cardiology, Democritus University of Thrace, Alexandroupolis, Greece
We conducted a retrospective analysis extracting the data from Google Trends ${ }^{\mathrm{TM}}$ (https://trends.google.com) to explore the interest of Italian population on acute PE from the 1st March to 13th September 2020 using the term "Pulmonary Embolism" (translated in Italian: Embolia Polmonare). We started the analysis in March 2020, because the Italian government applied the general lockdown on March 3rd. Collecting the number of Google queries, we were able to generate and then analyze the trend over the entire study period. Data were extracted and processed according to Nuti et al. [4]. Data of daily deaths for COVID-19 patients were retrieved by the Italian ministry of health and then converted to weekly rates.

As previously described, GT presents the results as relative search volume (RSV) divided for different geographical areas over a specific period. More specifically, RSV ranges from 0 to 100: the former value indicates no interest in the issue whereas the latter represents the peak search volume [5]. Ethics approval for this type of study was not required as none of the queries in the Google database can be associated with any identity and/or physical location, as specified in Google's privacy policy (www.google.com/privacypol icy.html).

Weekly RSVs were expressed as mean whereas temporal trends over the study period were analysed using the Jonckheere-Terpstra trend test. GT data were also compared with the number of weekly deaths of COVID-19 patients. Statistical significance was defined as $\mathrm{p}<0.05$. Statistical analysis was performed using SPSS package version 20.0 (SPSS, Chicago, Illinois, USA). Time-lag correlation analysis was performed using $\mathrm{R}$.

The number of patients dying with COVID-19 infection in Italy rose sharply through March 2020 (orange line), and during the same month the relative search frequency of the terms PE nearly doubled (increase of 56.3\% per week, red dashed line) (Fig. 1a). A strong correlation between the number of COVID-19-related deaths and Google search queries for PE (Pearson correlation coefficient 0.75 with 
Fig. 1 Mean weekly Italian relative search volume (RSV) for the item 'Pulmonary embolism' (blue line) and COVID-19 death (orange line) over the study period.
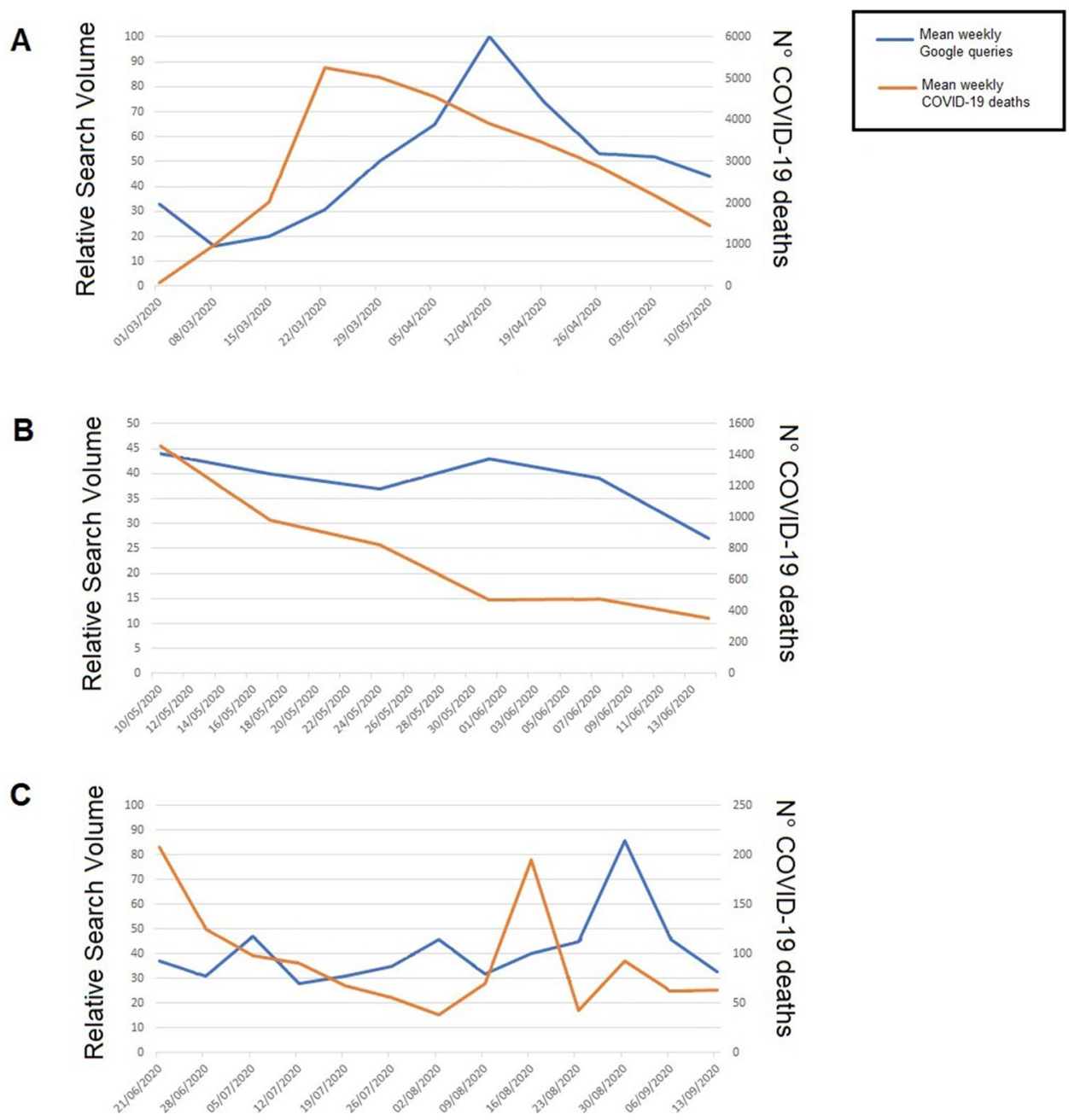

20-day time-lag) was also identified. With the reduction of restrictive measures applied by the Italian Government, both the number of COVID-19 deaths and the interest in acute PE decreased (the latter decreasing by $33.4 \%$ per week) (Fig. 1b). Conversely, during the so called "second phase" of the pandemic, the interest on acute PE rose again $(+21.4 \%$ per week) despite a relatively stable number of COVID-19 deaths (Fig. 1c).

Our results demonstrate a strong correlation between the increase in COVID-19 deaths and the use of Google to obtain information on acute PE, which has been presented as a frequent complication of Sars-CoV-2 infection. Probably, the media coverage of the issue raised the need for more information among the Italian population, also because Italy was the first western country severely affected by the COVID-19 pandemic. In addition, the fear of infection could have led the general population to use internet search, ignoring that PE was generally a complication of the disease and not a first manifestation of the infection. We can also not exclude that the lockdown period might have generated a higher incidence of venous thromboembolism (VTE) by favouring immobilization. In this regard, national data on the epidemiology of VTE during that period are not yet available, so further analyses will be necessary to investigate this issue. The increase interest on acute PE could be also due to the "curiosity" of general population on this disease in an attempt to early recognize suggestive symptoms. In this regard, a similar use of GT has been already reported during the COVID-19 pandemic for gastrointestinal symptoms [6] and anosmia [7].

Doubtless, our analysis has some limitations. First, our data are derived from only one Internet search engine; however, it has been reported that Google is the most popular search engine processing. Second, we could not discriminate the individuals simply interested in acute PE from those actually affected by disease. Finally, we cannot exclude that some mediatic events reporting the association between COVID-19 and PE could have influenced the number of Google queries.

In conclusion, our brief study demonstrated a higher interest in acute PE among Italian population during the lockdown period. This positive trend seems to indicate a 
substantial influence of social media in disseminating medical knowledge and influencing the web search in the Italian population.

Funding None.

\section{Compliance with ethical standards}

Conflicts of interest S.B. reports personal fees from Biocompatibles Group UK and Bayer HealthCare, non-financial support from Bayer HealthCare and Daiichi Sankyo, and institutional grants from Sanofi, outside the submitted work. S.V.K. reports grants and non-financial support from Bayer AG; grants and personal fees from Boehringer Ingelheim, personal fees from Bayer AG, grants and personal fees from Actelion, grants and personal fees from Daiichi-Sankyo, grants and personal fees from Biocompatibles Group UK, personal fees from Pfizer-Bristol-Myers Squibb, grants and personal fees from MSD, outside the submitted work. The other authors have no conflicts of interest to report.

\section{References}

1. Roncon L, Zuin M, Barco S, Valerio L, Zuliani G, Zonzin P, Konstantinides SV (2020) Incidence of acute pulmonary embolism in COVID-19 patients: Systematic review and meta-analysis. Eur J
Intern Med. S0953-6205(20):30349-6. https://doi.org/10.1016/j. ejim.2020.09.006

2. Islam MS, Sarkar T, Khan SH, Mostofa Kamal AH, Hasan SMM, Kabir A, Yeasmin D, Islam MA, Amin Chowdhury KI, Anwar KS, Chughtai AA, Seale H (2020) COVID-19-related infodemic and its impact on public health: a global social media analysis. Am J Trop Med Hyg. https://doi.org/10.4269/ajtmh.20-0812

3. Sousa-Pinto B, Anto A, Czarlewski W, Anto JM, Fonseca JA, Bousquet J (2020) Assessment of the impact of media coverage on COVID-19-related google trends data: infodemiology study. J Med Internet Res 22:e19611

4. Nuti SV, Wayda B, Ranasinghe I, Wang S, Dreyer RP, Chen SI, Murugiah K (2014) The use of google trends in health care research: a systematic review. PLoS One 9:e109583

5. Zuin M, Rigatelli G, Ronco F (2020) Worldwide and european interest in the mitraclip: a google trends-based analysis. J Cardiovasc Med (Hagerstown) 21:246-249

6. Rajan A, Sharaf R, Brown RS, Sharaiha RZ, Lebwohl B, Mahadev $S$ (2020) Association of search query interest in gastrointestinal symptoms With COVID-19 diagnosis in the united states: infodemiology study. JMIR Public Health Surveill 6:e19354

7. Walker A, Hopkins C, Surda P (2020) Use of google trends to investigate loss-of-smell-related searches during the COVID-19 outbreak. Int Forum Allergy Rhinol 10:839-847

Publisher's Note Springer Nature remains neutral with regard to jurisdictional claims in published maps and institutional affiliations. 